\title{
Croissance, morphogenèse et dynamique de l'état physiologique des bourgeons de jeunes plants de Châtaignier (Castanea sativa Miller) en conditions naturelles et contrôlées
}

\author{
Yvonne PEZET et Chadouli SI-MOHAMED \\ Université Blaise Pascal, Laboratoire de Phytomorphogenèse \\ U.A.-45 C.N.R.S., 4, rue Ledru, F 63038 Clermont-Ferrand Cedex
}

\begin{abstract}
Résumé
En conditions de culture constantes (température, photopériode), le Châtaignier manifeste un rythme de croissance endogène caractérisé par une alternance de périodes d'activité et de repos, d'autant plus irrégulières que la température est élevée.

L'expression de ses potentialités morphogénétiques apparaît fortement influencée par la température. Si dans la nature le Châtaignier est une espèce sympodiale à port arborescent, en conditions de culture constantes et à température élevée $\left(25^{\circ} \mathrm{C}\right.$ en jour long), la chute du bourgeon terminal (sympode), associée à une diminution des influences exercées par l'axe sur les bourgeons, favorise l'expression de la basitonie et le port buissonnant. Une température fraîche $\left(12^{\circ} \mathrm{C}\right.$ en jour long), par contre, favorise la formation d'un bourgeon terminal écailleux (monopode), empêche la prise de préséance des bourgeons du collet et maintient l'acrotonie de croissance qui conditionne un port arborescent.

En conclusion, une approche biochimique est proposée pour caractériser les différences d'aptitude à la croissance des bourgeons selon leur position sur l'axe et selon les conditions d'environnement.
\end{abstract}

Mots clés: Châtaignier, morphogenèse, repos, rythme, température.

\section{Introduction}

Le Châtaignier (Castanea sativa Miller) occupe une place particulière parmi les essences forestières françaises. En effet, malgré l'importance des surfaces qu'il occupe (560000 ha), l'intérêt porté à sa production fruitière (Invuflec, 1975 ; Bergougnoux et al. 1978) ou sylvicole (CAmus, 1929 ; DE Champs, 1972), c'est à la fois une espèce familière et méconnue. En particulier, les premiers stades de sa croissance n'ont pas fait l'objet d'études précises bien qu'ils s'avèrent importants pour le développement ultérieur du végétal.

Seuls Lavarenne et al. (1971) ont abordé une telle étude en condition phytotronique et souligné l'existence d'une croissance rythmique à $25^{\circ} \mathrm{C}$ en jour long, avec affaiblissement progressif de la dominance de l'axe épicotylé et naissance de ramifica- 
tions vigoureuses à la base de l'axe, ce qui n'est jamais observé en conditions naturelles.

Le présent article propose une analyse précise de la croissance et de la morphogenèse de jeunes Châtaigniers issus de semis, en fonction des conditions climatiques naturelles ou réalisées en chambres de culture. Pour interpréter les comportements observés dans les différentes situations étudiées, nous avons été conduits à apprécier l'état physiologique des bourgeons en fonction de leur position sur la plante, au moyen d'un test biologique classique ; celui-ci consiste à mesurer l'aptitude à la croissance d'une population de bourgeons isolés portés par un fragment d'axe.

Cette technique a permis de mettre en évidence l'existence d'un gradient médiobasitone d'aptitude au débourrement le long de l'axe pendant le repos hivernal ( $\mathrm{S}_{\mathrm{I}}$ Mohamed, 1983). Ce gradient s'inverse plusieurs semaines avant la reprise de croissance, comme chez de nombreuses espèces arborescentes (CrabBé, 1968 ; Barnola, 1976 (b) ; Jourdan, 1980 ; ZANETTE, 1981).

Dans le présent article nous analysons le rôle de la température sur la conservation ou la modification de ces gradients et leurs conséquences morphogènes.

\section{Matériel et techniques}

\subsection{Matériel}

Des semences de Châtaignier, Castanea sativa Miller, sont récoltées sous un même arbre dans la région de Durtol (Puy-de-Dôme) à $600 \mathrm{~m}$ d'altitude. Les semences les plus volumineuses (13 à $15 \mathrm{~g}$ ) sont mises à tremper dans l'eau pendant une semaine. puis dans une solution contenant $0,4 \mathrm{~g} \cdot \mathrm{l}^{-1}$ de benomyl et $1 \mathrm{~g} \cdot 1^{1}$ de méthylthiophanate ; après ressuyage et enrobage par le soufre $\left(2 \mathrm{~g} \cdot \mathrm{kg}^{-1}\right)$, elles sont stockées dans des bacs contenant de la vermiculite, en chambre obscure à $4{ }^{\circ} \mathrm{C}$.

\subsection{Techniques}

\subsection{Culture}

a) En conditions naturelles

Une cinquantaine de semences germées sont mises en culture, en pleine terre, en avril 1981, à Clermont-Ferrand $(374 \mathrm{~m})$. En période sèche les plants sont régulièrement arrosés.

\section{b) En chambres climatisées}

Une cinquantaine de semences germées sont mises en culture en conteneurs (3 1) contenant un mélange équivolumique de tourbe brune, de pouzzolane et de terre de Châtaigneraie. Les plants sont arrosés une fois par semaine avec une solution nutritive (LAMOND, 1978) et chaque fois qu'il est nécessaire avec de l'eau du robinet. Ils reçoivent un éclairement de 100 à $150 \mathrm{~W} \cdot \mathrm{m}^{-2}$ mesuré à $50 \mathrm{~cm}$ du plafond lumineux, dispensé par des tubes fluorescents (de type lumière du jour de luxe, $60 \mathrm{~W}$ ) et par des 


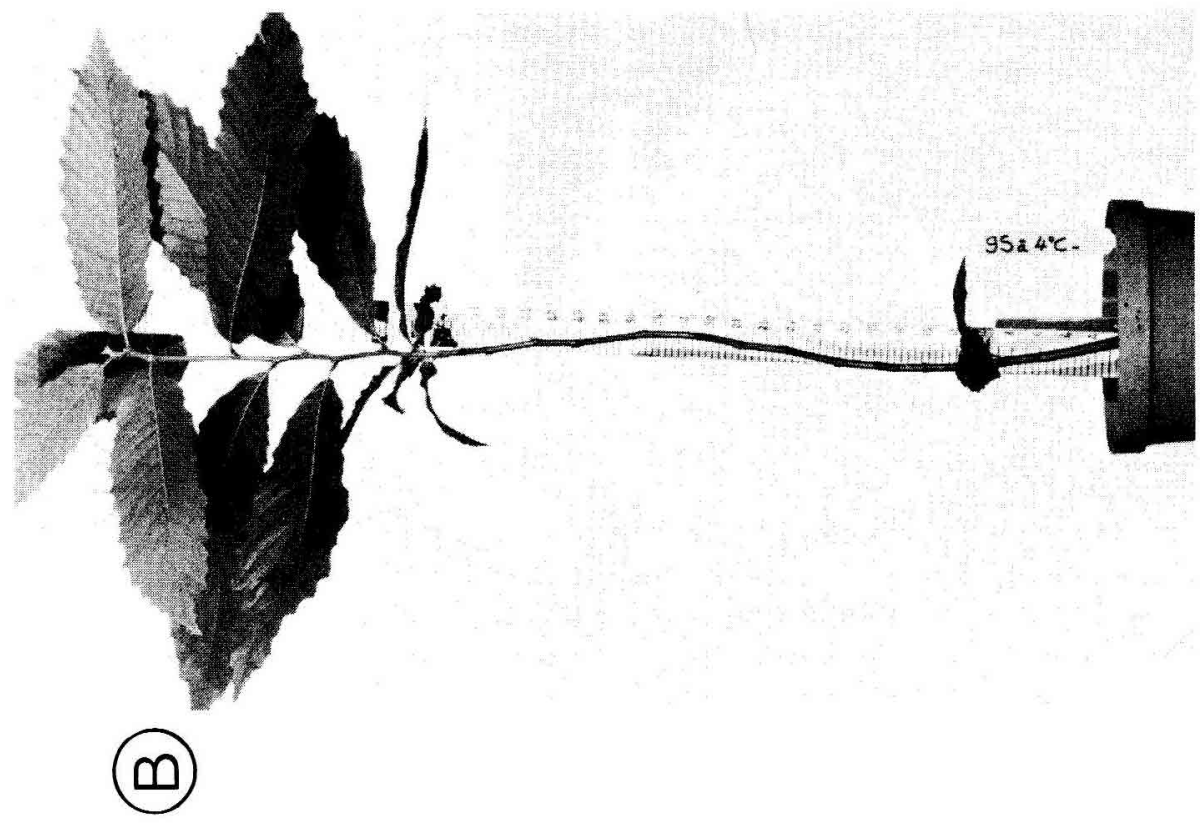

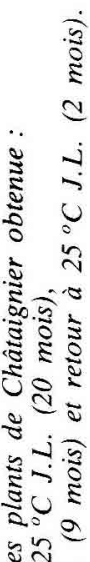

章分-

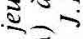

(1)

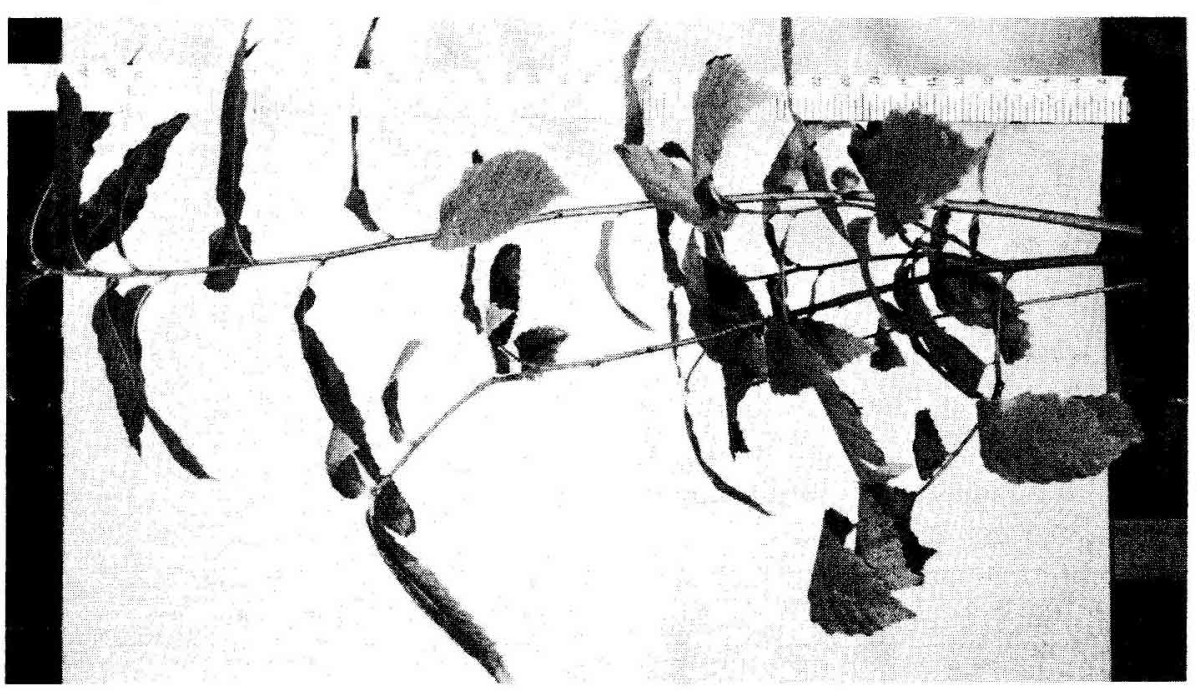

$\approx \leq 0$

.7

$\frac{2}{\frac{2}{2}}$ 
lampes à incandescence. Deux conditions de culture ont particulièrement retenu notre attention; il s'agit de $25^{\circ} \mathrm{C}$ et de $12^{\circ} \mathrm{C}$ en jour long (J.L. : $16 \mathrm{~h}$ de lumière pour $8 \mathrm{~h}$ d'obscurité), en raison du rôle morphogène différent de ces deux régimes thermiques.

\subsection{Paramètres étudiés et expression des résultats}

La croissance en longueur et le nombre moyen de feuilles apparues sont enregistrés chaque semaine pendant au moins 3 cycles de végétation. L'allongement et le rythme plastochronique apparent sont exprimés sous forme de diagrammes en barres.

\subsection{Analyse de l'état physiologique des bourgeons : le test des boutures de nouds isolés}

\section{a) Principe}

Ce test permet d'apprécier la plus ou moins grande aptitude à la croissance d'une population de bourgeons portés par un fragment d'axe court, placés sur un substrat composé de tourbe et de sable et mis en culture dans un large éventail thermique (Pouger, 1963). Nous considérons comme Vegrs (1964) qu'un bourgeon est d'autant plus dormant que la gamme des températures dans laquelle il peut croître est plus restreinte.

Cette technique n'est pas exempte de critiques (Rageau, 1975 ; Barnola, 1976 (b) ; Champagnat, 1983 ; Regnard, 1984), mais elle reste encore aujourd'hui un outil précieux d'analyse et de compréhension de la morphogenèse des végétaux ligneux.

\section{b) Mise en cuvre de la technique et expression des résultats du test}

Des boutures de nœuds isolés d'environ $5 \mathrm{~cm}$ de longueur sont prélevées à 3 niveaux du végétal : apical (portant le bourgeon terminal ou subterminal), médian et au niveau du collet (portant les bourgeons cotylédonaires). Les boutures, paraffinées à leur extrémité supérieure pour limiter le dessèchement, sont placées verticalement dans des boîtes contenant de la tourbe humide traitée au benomyl $\left(1 \mathrm{~g} \cdot \mathrm{l}^{-1}\right)$. Les boîtes contenant 15 boutures sont placées en chambres climatisées à $(12 \pm 1),(18 \pm 1)$ et $(25$ $\pm 2)^{\circ} \mathrm{C}$ en jour long.

Le test est réalisé sur des lots de plants élevés dans les conditions suivantes : dans la nature, en chambres climatisées à $25^{\circ} \mathrm{C} \mathrm{J}$.L. et à $4{ }^{\circ} \mathrm{C}$ J.L. Ce dernier traitement est imposé à des Châtaigniers dès l'entrée en repos à $25^{\circ} \mathrm{C}$ J.L. A cette condition, les feuilles chutent après 4 à 6 semaines.

Les plants élevés dans les conditions naturelles sont prélevés tous les 15 jours depuis mi-septembre jusque mi-mars. L'état physiologique des bourgeons subterminaux est comparé à celui des bourgeons médians et cotylédonaires. Pour les plants er repos à $25^{\circ} \mathrm{C}$ J.L. ou à $4{ }^{\circ} \mathrm{C}$ J.L., des lots de 15 plants sont fragmentés en boutures toutes les 3 semaines pendant 6 mois. L'évolution comparée des bourgeons subterminaux, médians et cotylédonaires est étudiée à $25^{\circ} \mathrm{C}$ J.L.

Le paramètre choisi pour caractériser l'état de dormance des bourgeons prélevés à une date donnée est le pourcentage maximum de débourrement observé au bout de 40 jours dans les conditions du test. Le stade du débourrement est caractérisé par l'écartement des premières paires d'écailles accompagnant le gonflement et l'allongement du bourgeon. 


\section{Résultats}

\subsection{Croissance et morphogenèse du Châtaignier en conditions naturelles}

\subsection{La croissance}

Les jeunes plants de Châtaignier présentent une croissance rythmique. Elle est caractérisée dès le premier cycle de végétation par 2 grandes vagues de croissance (fig. 1). La première a lieu du début du printemps au début de l'été et dure en moyenne 2 à 3 mois. La deuxième pousse peut être soit plus brève et moins vigoureuse que la première (en 1981, elle mesure $4 \mathrm{~cm}$ et porte 7 feuilles tandis que la première pousse mesure $19 \mathrm{~cm}$ et porte 10 feuilles) soit plus longue et plus vigoureuse que la première ou de même dimension (en 1982, elle mesure $37 \mathrm{~cm}$ et porte 18 feuilles alors que la première pousse ne mesure que $27 \mathrm{~cm}$ et porte 18 feuilles).

L'arrêt de la croissance (fín août) est marqué par le dessèchement et la chute de l'extrémité terminale, c'est-à-dire du bourgeon terminal accompagné de l'entre-nœud sous-jacent dégagé. La croissance est donc sympodiale et acrotone dès les premiers cycles de végétation.

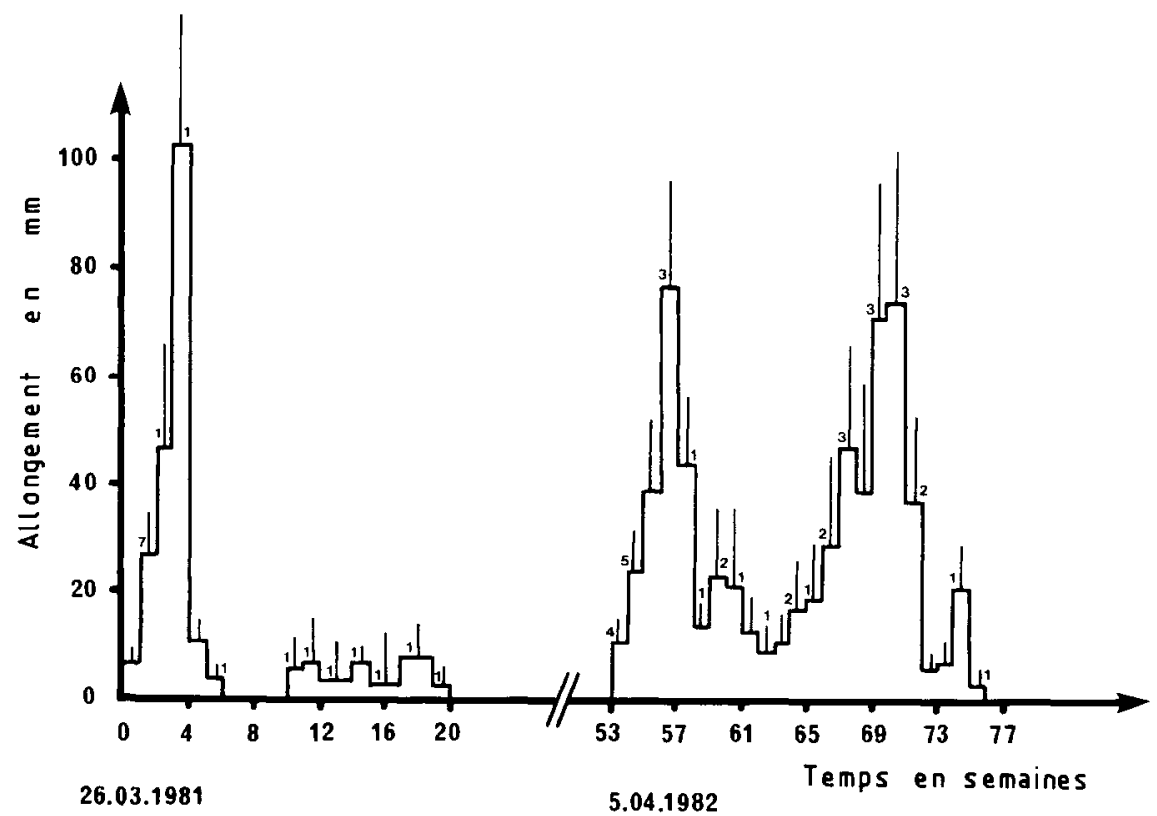

FIG. 1

Allongement hebdomadaire de l'axe principal de plants de Châtaignier, issus de semis, dans la nature (à partir du $2^{e}$ cycle de végétation).

Le nombre de feuilles apparues est indiqué sur chaque colonne du diagramme.

Un tiret symbolise l'intervalle de confiance au seuil 5 p. 100.

Weekly stem elongation of young Chestnut seedlings after germination, in natural conditions.

Vertical bars represent standard deviation of mean, at 5 p. 100.

The number of outspread leaves is reported above each diagram column. 


\subsection{Caractérisation de l'état de dormance des bourgeons}

Le test des boutures de nœuds isolés fait apparaître une forte «inertie » au débourrement des bourgeons subterminaux au cours des mois de septembre et octobre (fig. 2). A cette période, aucun bourgeon n'évolue à $12{ }^{\circ} \mathrm{C}$ et à $18^{\circ} \mathrm{C}$. A $25^{\circ} \mathrm{C}$, une croissance est possible et le taux maximum de débourrement passe de 20 à $100 \mathrm{p}$. 100 entre fin septembre et mi-novembre. L'inertie au débourrement s'estompe au cours de l'automne et fin janvier au moins 65 p. 100 des bourgeons subterminaux débourrent à la température de $12{ }^{\circ} \mathrm{C}$.

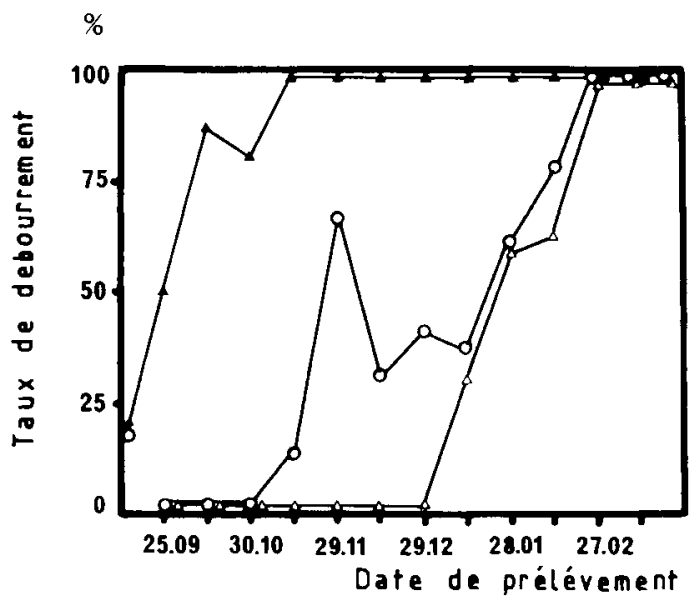

Fici. 2

Evolution du taux maximum de débourrement des bourgeons subterminaux,

en boutures de nauds isolés, en fonction de la date de prélèvement dans la nature.

Le test est réalisé à $(12 \pm 1)^{\prime \prime} \mathrm{C}(\triangle-\triangle) .(18 \pm 1)^{\circ} \mathrm{C}(\mathrm{O}-\mathrm{O})$ et $(25 \pm 2)^{\circ} \mathrm{C}(\mathbf{\Delta}-\mathbf{\Delta})$ en jour long.

Evolution of maximal rate of subapical bud burst, on isolated node cuttings, with sampling date and under natural conditions. Test was carried out under 3 controlled temperatures : $(I 2 \pm I)^{\circ} \mathrm{C}(\Delta-\Delta),(18 \pm I)^{\prime \prime} \mathrm{C}(\mathrm{O}-\mathrm{O})$ and $(25 \pm 2)^{\circ} \mathrm{C}(\Delta-\Delta)$ in long days.

En septembre, les bourgeons subterminaux se développent moins facilement à 25 " $\mathrm{C}$ que les bourgeons médians et cotylédonaires (fig. 3). Ils débourrent en moins grand nombre et avec un temps de latence plus long. Dès fin octobre, ce gradient médio-basitone d'aptitude au débourrement disparaît, et à la fin de l'hiver une partie des bourgeons du collet ne peuvent plus croître en boutures de nouds isolés. Le froid automno-hivernal permet donc l'élargissement de la gamme des températures compatibles avec le débourrement, élimine le gradient médio-basitone d'aptitude au débourrement installé à la fin de l'été et permet le rétablissement de la préséance apicale (acrotonie de débourrement).

\subsection{Croissance et morphogenèse du Châtaignier cultivé à température élevée} et constante : $25^{\circ} \mathrm{C}$ J.L.

\subsection{La croissance}

La première vague de croissance dure environ 22 semaines (fig. 4). Les plantes mesurent en moyenne $60 \mathrm{~cm}$ et portent environ 26 feuilles. L'arrêt de la croissance se 


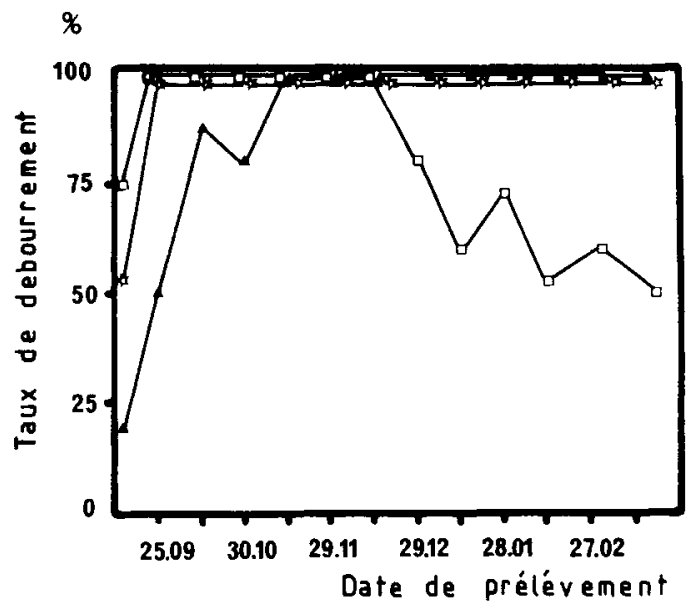

FIG. 3

Evolution comparée du taux maximum de débourrement des bourgeons subterminaux $(\boldsymbol{\Lambda}-\mathbf{\Delta})$, médians ( $\vec{z}-\vec{j}$ ) et cotylédonaires $(\square-\square)$, en boutures de nouds isolés, à $(25 \pm 2)^{\circ} \mathrm{C} J . L$., en fonction de la date de prélèvement dans la nature.

Comparative evolution of maximal rate of bud burst for subapical ( $\mathbf{\Delta}-\mathbf{\Delta})$, middle (it - is). and cotyledonary buds $(\square-\square)$, on isolated node cuttings, with sampling date under natural conditions.

Test was carried out at $(25 \pm 2)^{\circ} \mathrm{C}$.

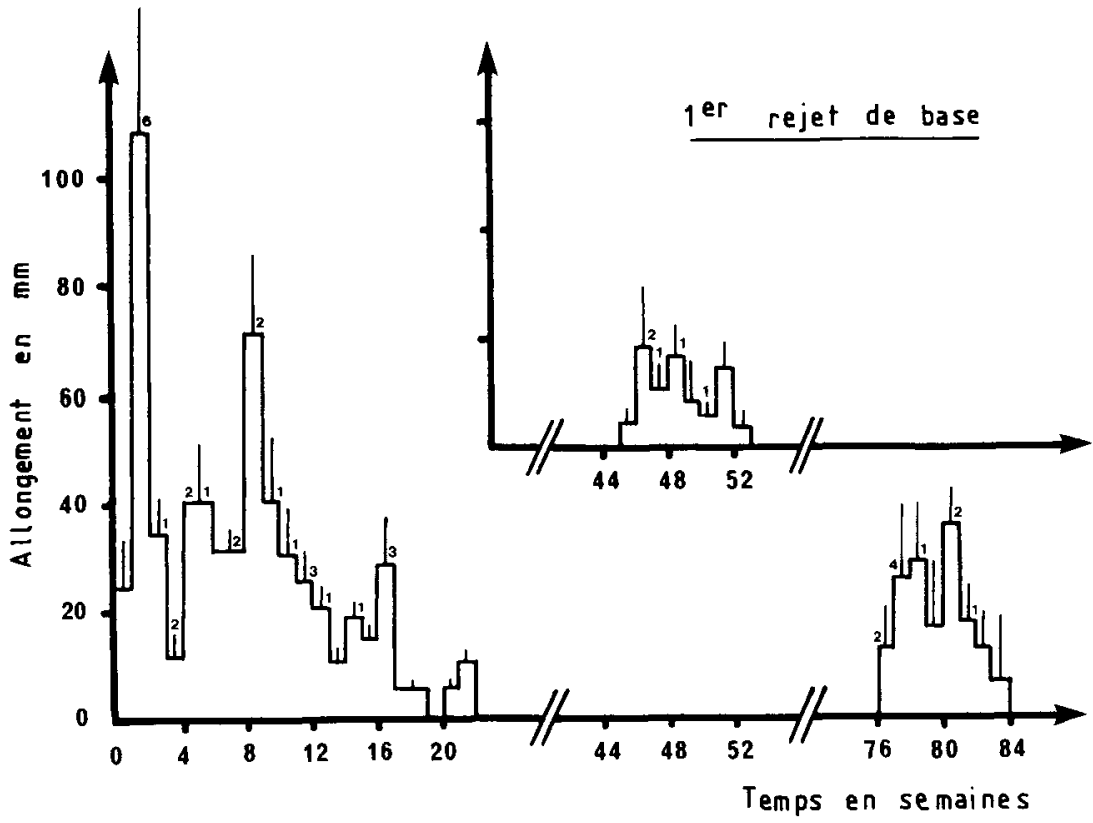

Fig. 4

Allongement hebdomadaire de plants de Châtaignier, issus de semis, cultivés en conteneurs $\grave{a}(25 \pm 2)^{\circ} \mathrm{C}$ en jour long, pendant 2 ans.

Le nombre de feuilles apparues est indiqué sur chaque colonne du diagramme.

Un tiret symbolise l'intervalle de confiance au seuil 5 p. 100.

Weekly stem elongation of young Chestnut seedlings after germination, at $(25 \pm 2)^{\circ} \mathrm{C}$ in long days, during 2 years.

The number of outspread leaves is reported above each diagram column.

Vertical bars represent standard deviation of mean, at 5 p. 100. 
caractérise par le ralentissement brutal de l'allongement puis le dessèchement et la chute de l'extrémité terminale du plant. La croissance est sympodiale dès le premier cycle de végétation. 6 mois après cet arrêt, ce sont les bourgeons basaux et surtout cotylédonaires qui engendrent les pousses les plus longues (environ $75 \mathrm{~mm}$ avec une moyenne de 5 feuilles). Les bourgeons subterminaux débourrent quelquefois, mais ne produisent pas de véritables pousses.

Après 1 an de culture à $25^{\circ} \mathrm{C}$, la morphologie des plants est de type buisson.

La troisième vague de croissance survient 6 mois après l'arrêt de croissance des bourgeons basaux. Elle se caractérise par le débourrement des bourgeons insérés sur les $2 / 3$ supérieurs de la plante. Le bourgeon subterminal engendre la pousse la plus longue (152 $\mathrm{mm}$ avec 10 feuilles) et croît durant 8 semaines environ, tandis que les bourgeons médians donnent naissance à des pousses courtes mesurant, au bout de 2 à 4 semaines d'allongement, environ $30 \mathrm{~mm}$ et portant 4 feuilles.

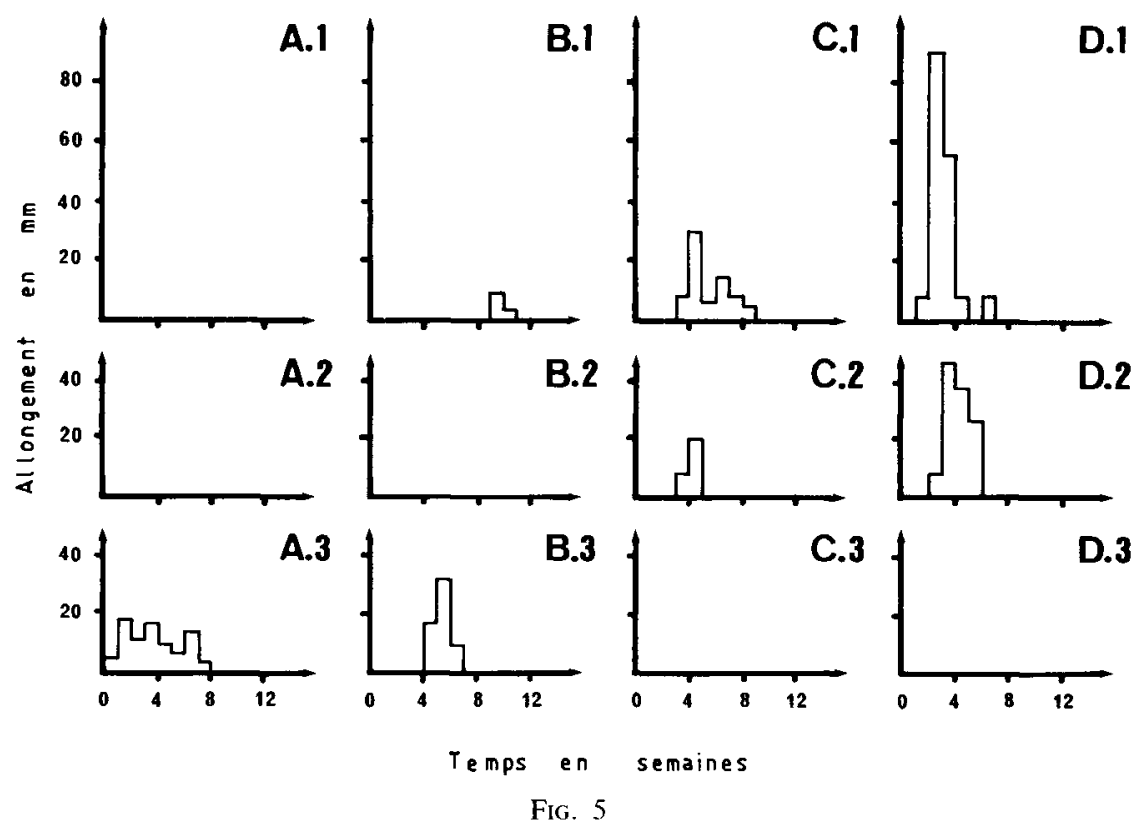

Effet de traitement à $4^{\circ} \mathrm{C} \mathrm{J.L.} \mathrm{sur} \mathrm{la} \mathrm{reprise} \mathrm{de} \mathrm{croissance} \mathrm{à} 25^{\circ} \mathrm{C} \mathrm{J.L.} \mathrm{des} \mathrm{bourgeons} \mathrm{subterminaux} \mathrm{(1),}$ médians (2) et cotylédonaires (3) de lots de 10 plants.

A. Plants témoins restés à $25^{\circ} \mathrm{C}$ J.L. :

le temps 0 correspond au débourrement des bourgeons cotylédonaires.

B. Plants traités 6 semaines à $4^{\circ} \mathrm{C}$ J.L., dès le repos à $25^{\circ} \mathrm{C}$ J.L.

C. Plants traités 12 semaines à $4^{\circ} \mathrm{C}$ J.L., dès le repos à $25^{\circ} \mathrm{C}$ J.L.

D. Plants traités 24 semaines à $4^{\circ} \mathrm{C}$ J.L., dès le repos à $25^{\circ} \mathrm{C} \mathrm{J.L.}$

Effect of cooling at $4^{\circ} \mathrm{C}$ on the opening of subapical (I), middle (2) and cotyledonary buds (3), at $25{ }^{\circ} \mathrm{C}$ in long days, for 10 plants.

A. Control plants maintained at $25^{\circ} \mathrm{C}$ L.D. : time scale begins with cotyledonary bud burst.

B. Treated plants, 6 weeks at $4^{\circ} \mathrm{C}$ L.D., after the cessation of shoot growth.

C. Treated plants, 12 weeks at $4^{\circ} \mathrm{C}$ L.D., after the cessation of shoot growth.

D. Treated plants, 24 weeks at $4^{\circ}$ C L.D., after the cessation of shoot growth. 
Bien que quelques bourgeons médians puissent se développer, cette $3^{\text {e }}$ vague de croissance est marquée par le rétablissement de l'acrotonie. A $25^{\circ} \mathrm{C}$ J.L., le Châtaignier est donc alternativement acrotone ( $1^{\text {re }}$ et $3^{\text {e }}$ vague de croissance) et basitone ( $2^{e}$ vague).

\subsection{Caractérisation du repos apparent}

a) Traitements capables de rétablir la croissance apicale après la première vague de croissance

Plusieurs moyens peuvent être employés pour réactiver la croissance du bourgeon subterminal des plants en repos à $25^{\circ} \mathrm{C}$ J.L. L'ablation du feuillage n'est plus efficace au-delà de 4 semaines après l'entrée en repos apparent ; les transplantations, l'apport minéral ou hormonal (BAP à $1 \mathrm{~g} \cdot \mathrm{l}^{-1}$ ) ne permettent pas d'éliminer l'amortissement du rythme de croissance qui intervient inéluctablement chez le Châtaignier.

Par contre, l'exposition des plants au froid $\left(4{ }^{\circ} \mathrm{C}\right.$ J.L.) pendant 6 semaines au moins, dès l'installation du repos apparent à $25^{\circ} \mathrm{C}$ J.L., permet de rétablir la préséance apicale. Après retour à $25^{\circ} \mathrm{C} \mathrm{J}$.L., la croissance est de nouveau acrotone après un temps de latence d'autant plus court que le traitement au froid a été plus long (fig. 5). L'acrotonie est d'autant plus forte que la durée du séjour à $4{ }^{\circ} \mathrm{C}$ a été plus grande (de 9 à 24 semaines). Les bourgeons basaux et cotylédonaires sont alors inaptes à la croissance.

b) Caractérisation de l'état de dormance des bourgeons

Le test des boutures de nouds isolés révèle qu'au terme de la première vague de croissance, il s'installe à l'échelle du plant entier un gradient médio-basitone d'aptitude au débourrement (fig. 6). Les bourgeons de la partie terminale de l'axe présentent une

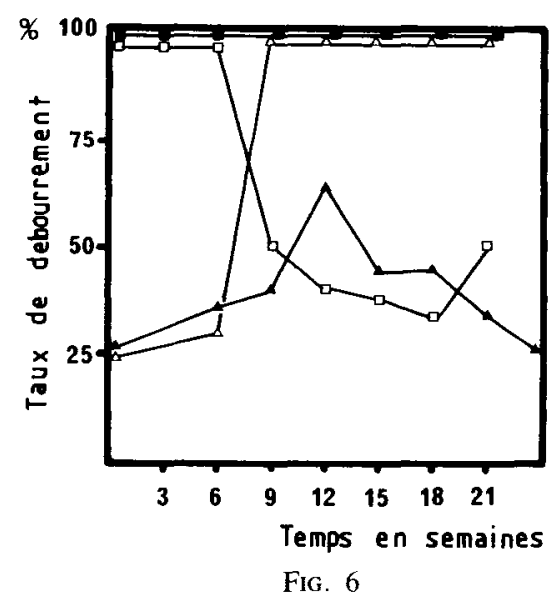

Evolution du taux maximum de débourrement des bourgeons subterminaux ( $\Delta$ et $\triangle$ )

et cotylédonaires $\left(\square\right.$ et $\square$ ) en boutures de nouds isolés, à $25^{\circ} \mathrm{C}$ en jour long.

Comparaison des bourgeons issus de plants à $25^{\circ} \mathrm{C}$ J.L. ( $\mathbf{\Delta}$ et $\left.\mathbf{\square}\right)$ et $\grave{a} 4{ }^{\circ} \mathrm{C} \mathrm{J.L.}(\triangle$ et $\square)$ en fonction du délai suivant le repos.

Evolution of maximal rate of bud burst for subapical ( $\Delta$ and $\triangle$ ) and cotyledonary buds ( $\square$ and $\square$ ) on isolated note cuttings, at $25^{\circ} \mathrm{C}$ L.D.

Comparative evolution of buds coming from plants at $25^{\circ} \mathrm{C}$ L.D. (A and $\left.\mathbf{0}\right)$ and at $4^{\circ} \mathrm{C}$ L.D. ( $\triangle$ and $\square$ ), according to time following cessation of shoot growth. 
forte inertie à la croissance caractérisée par un taux de débourrement faible (25 à 60 p. 100 maximum) et un temps de latence au débourrement important (12 à 16 jours) pendant toute la période de repos apparent à $25^{\circ} \mathrm{C}$ J.L., tandis que les bourgeons de la partie proximale sont toujours aptes à croître.

Après un traitement à $4{ }^{\circ} \mathrm{C}$ pendant 6 à 9 semaines, il n'existe pratiquement plus de gradient d'aptitude au débourrement: tous les bourgeons de l'axe débourrent en même temps. Au-delà de 9 semaines d'exposition au froid, le gradient d'aptitude au débourrement est nettement acrotone : le taux maximum de débourrement des bourgeons cotylédonaires n'atteint plus 50 p. 100.

\subsection{Croissance et morphogenèse de jeunes plants de Châtaignier cultivés à température fraîche et constante : $12{ }^{\circ} \mathrm{C}$ J.L.}

La première vague de croissance dure de 12 à 15 semaines (fig. 7). Les plants mesurent alors en moyenne $19 \mathrm{~cm}$ et portent 10 feuilles. La deuxième vague de croissance survient à peu près 5 mois après le repos et dure environ 8 semaines. La pousse obtenue mesure en moyenne $62 \mathrm{~mm}$ et porte 7 feuilles. La troisième vague de croissance débute environ 6 mois après l'arrêt de la vague précédente; elle dure 8 semaines et la pousse mesure alors $26 \mathrm{~mm}$ et porte 5 feuilles.

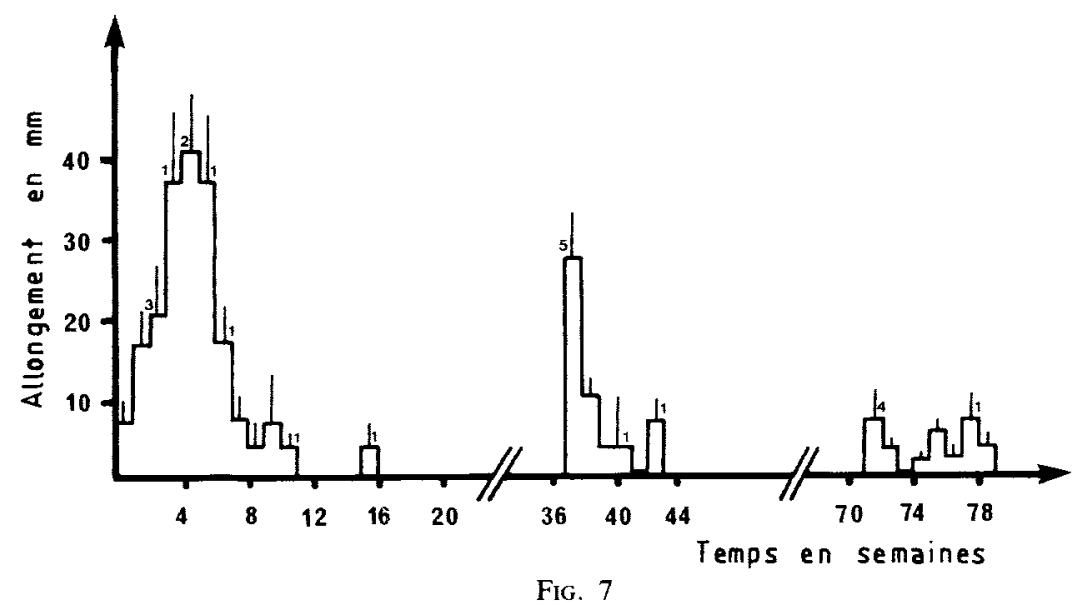

Allongement hebdomadaire de l'axe principal de plants de Châtaignier, issus de semis, cultivés en conteneurs à $(12 \pm 1)^{\circ} \mathrm{C}$, en jour long.

Le nombre de feuilles apparues est indiqué sur chaque colonne du diagramme.

Un tiret symbolise l'intervalle de confiance au seuil de $5 \mathrm{p} .100$.

Weekly stem elongation of young Chestnut seedlings after germination, at $(12 \pm 1)^{\circ} \mathrm{C}$ in long days.

The number of outspread leaves is reported above each diagram column.

Vertical bars represent standard deviation of mean, at 5 p. 100.

La fin de chaque vague de croissance est caractérisée par la formation d'un bourgeon terminal écailleux.

Dans ces conditions de température, la croissance est monopodiale et strictement acrotone. 


\section{Discussion}

\subsection{Rythme de croissance et environnement}

En conditions naturelles, le Châtaignier présente une croissance rythmique que modulent les facteurs du milieu. Il partage ce caractère avec la plupart des arbres de climats tempérés (LAVAREnNe et al., 1971 ; Champagnat et al., 1986) et de climats tropicaux (Hallé et Oldemann, 1970 ; Parisot, 1985). Contrairement à une population de Hêtres dont chaque individu présente un comportement aléatoire (les uns pouvant cesser de croître précocement, alors que d'autres présentent deux vagues de croissance d'intensité équivalente (RIEDACKER, 1981)), une population de Châtaigniers a un comportement relativement homogène. Tous les individus d'une population, issus d'un lot de semences homogènes (poids, calibre), présentent une deuxième vague de croissance d'importance et de durée équivalentes. Toutefois les caractéristiques de cette deuxième vague peuvent varier d'une année sur l'autre en fonction des facteurs climatiques.

La culture en condition climatique constante, en jour long ou continu et à température élevée, met en évidence comme pour le Chêne et le Hêtre (Lavarenne et al., 1971 ; Champagnat et al., 1986) le caractère endogène du rythme de croissance du Châtaignier.

Quelle que soit la condition de culture, la première vague de croissance est plus vigoureuse et durable que les suivantes. Ce caractère serait lié à la présence des cotylédons riches en réserves, comme le montrent différentes études chez le Chêne (LAmond, 1978 ; LeVerT, 1982 ; PAyAN, 1982) ainsi qu'un travail préliminaire chez le Châtaignier. Dans ces mêmes conditions de température, la troisième vague de croissance est aussi de moindre intensité que la seconde. Comme chez le Pommier (Zanetre, 1981) et contrairement au Chêne (PAYAN, 1982), l'expression rythmique de la croissance s'affaiblit. Cependant si l'on place à des températures différentes les tiges et les racines, on peut s'opposer efficacement à l'affaiblissement de ce rythme. Ainsi pour le Pommier, une température de $25^{\circ} \mathrm{C}$ pour la partie aérienne et une température de $12{ }^{\circ} \mathrm{C}$ pour les racines permettent une croissance rythmique régulière soutenue (ZANeTte, 1981). Pour le Châtaignier, les températures basses appliquées aux parties aériennes alors que les racines sont placées à température élevée, favorisent la régularité du rythme de croissance (Si-Mohamed, 1983).

A $25^{\circ} \mathrm{C}$ J.L. un rythme de développement d'une autre nature peut être envisagé, si on considère les vagues de croissance successives assurées alternativement par les bourgeons du collet et les bourgeons subterminaux. Il s'agit d'un rythme de levée d'inhibition qui s'apparenterait davantage à celui décrit chez le Pêcher-amandier cultivé in vitro lors de l'apparition de rameaux anticipés (Guevara, 1984).

\subsection{Morphogenèse et températures}

Dans la nature, le gradient médio-basitone d'aptitude au débourrement révélé en boutures de nœuds isolés s'inverse ou s'annule à la fin de l'hiver chez tous les arbres étudiés jusqu'à présent : le Pommier (Crabbé, 1968 ; Zanetre, 1981), le Frêne ou le Tilleul (Barnola, 1976 (b)), le Peuplier (Jourdan, 1980) et le Noyer (Mauget, 1984). Le Châtaignier présente une dynamique d'évolution de la dormance semblable. Comme 
pour d'autres espèces ligneuses, c'est l'intégrité de l'axe qui conditionne la réalisation de l'acrotonie (Champagnat et al., 1971 ; Meng-HoRn et al., 1975 ; Barnola, 1976 (b)). Année après année cette acrotonie de croissance conduit à l'élaboration d'un tronc.

Ce comportement est à comparer à celui observé en conditions de culture constantes. A température élevée $\left(25^{\circ} \mathrm{C}\right.$ et aussi $\left.18^{\circ} \mathrm{C}\right)$ en jour long, le Châtaignier est une espèce chez laquelle alternent croissance acrotone et basitone (LAVARENNE et al., 1971 ; Barnola, 1976 (b) ; Si-Mohamed, 1983). Dans ces conditions de température la basitonie n'est pas réprimée ; elle peut au contraire s'exprimer et s'opposer à la mise en place d'un port arborescent. Les rejets de base se différencient à partir de zones méristématiques préexistantes dans la Châtaigne conservée à $4{ }^{\circ} \mathrm{C}$.

A $12{ }^{\circ} \mathrm{C}$, en jour long, par contre, le Châtaignier est strictement acrotone et les bourgeons du collet restent à l'état de zones méristématiques latentes. Ils ne deviennent jamais préséants malgré la longue période de repos du bourgeon terminal (5 à 6 mois).

Si la croissance monopodiale est caractéristique des plants cultivés à températures fraîches, la croissance sympodiale est propre à ceux cultivés à température élevée. Mais il n'y a pas de relation apparente entre le port arborescent ou buissonnant, et le monopole ou le sympode. En effet dans la nature, dès la première année, le Châtaignier est un jeune arbre sympodial. Des températures de culture différentes au niveau des tiges et des racines permettent d'obtenir soit un sympode si les racines sont exposées à $12{ }^{\circ} \mathrm{C}$ et les tiges à $25^{\circ} \mathrm{C}$, soit un monopode si au contraire les racines sont exposées à $25^{\circ} \mathrm{C}$ et les tiges à $12{ }^{\circ} \mathrm{C}$ (Si-Mohamed, 1983).

La température apparaît donc comme un facteur déterminant de la morphogenèse du Châtaignier. Cette espèce, ainsi que le Tilleul, se comporte comme un buisson de type Noisetier (Barnola, 1976 (a) et (b)) chez lequel les températures élevées permettent le buissonnement alors que les températures fraîches rétablissent la préséance apicale. Par contre il diffère des buissons comme le Sureau ou le Framboisier (BARNOLA, 1976 (b)), chez lesquels ce sont les températures basses qui permettent l'apparition de ramifications basitones, et de la plupart des arbres qui conservent leur port arborescent quelles que soient les conditions phytotroniques : Chêne, Frêne, Pommier, Manguier, etc. Dès lors, il apparaît comme un matériel d'étude privilégié pour approfondir le déterminisme de la formation d'un tronc ou d'un buisson.

\subsection{Corrélations et repos végétatif}

Dans la nature, le repos végétatif est caractérisé par un état de dormance faible et temporaire des bourgeons distaux du Châtaignier. Le froid automnal suffit à rétablir l'aptitude au débourrement de ces bourgeons en boutures de nouds isolés à $25^{\circ} \mathrm{C}$ ( $\mathrm{SI}$ MOHAMED, 1983).

Pendant le repos apparent à $25^{\circ} \mathrm{C}$, les bourgeons distaux présentent une inertie plus forte que les bourgeons du collet toujours aptes à débourrer en boutures de nœuds isolés, quelle que soit la température de réalisation du test. Les traitements permettant de retarder l'entrée en repos des plants cultivés à $25^{\circ} \mathrm{C}$ J.L., laissent supposer que la cessation d'activité des bourgeons terminaux est d'abord la conséquence de corrélations d'origine axiale, relayées ensuite par des inhibitions d'origine foliaire et finalement des territoires proches du méristème, comme c'est le cas du Poirier (HuET, 1965), de l'Hévéa (Halle et Martin, 1968), du Cacaoyer (Vogel, 1975), du Noyer (Mauget, 
1978) et du Chêne (PAYAN, 1982). La concurrence entre allongement et organogenèse accrue par les températures élevées caractériserait l'entrée en repos des bourgeons distaux de l'axe : le bourgeon terminal se nécrose après avoir produit de nombreux ensembles foliaires. Un traitement par le froid lèverait ces inhibitions et rétablirait la préséance apicale en même temps qu'il diminuerait les capacités de croissance des bourgeons du collet.

A $12{ }^{\circ} \mathrm{C}$ en jour long, le repos apparent serait caractérisé par une dormance vraie des bourgeons terminaux qui ne se nécrosent pas comme à $25^{\circ} \mathrm{C}$. Elle serait ensuite levée par la température qui l'a induite de la même façon que le Frêne ou le Noisetier. Pour ces espèces il a été montré l'importance du double mouvement d'entrée puis de sortie de dormance aux températures basses (LAVARENNE et al., 1975 et 1980 ; BARNOLA, 1976 (a) et (b) ; BARNOLA et al., 1977).

\section{Conclusions}

Quelles que soient les conditions de culture, la croissance du Châtaignier est caractérisée par une alternance de périodes d'activité et de périodes de repos d'autant plus irrégulières que la température de culture est plus élevée. Ce rythme a tendance à s'amortir en conditions de culture constantes et à température élevée, tandis que des températures fraîches $\left(4^{\circ} \mathrm{C}\right.$ ou $\left.12{ }^{\circ} \mathrm{C}\right)$ sont capables de s'opposer à l'affaiblissement du rythme comme le peuvent également les variations saisonnières des facteurs du milieu.

La température apparaît par ailleurs comme un facteur déterminant du contrôle de la morphogenèse du Châtaignier : les températures élevées permettent aux bourgeons du collet d'exprimer leur aptitude à la croissance, tandis que les températures fraîches favorisent l'édification d'un tronc.

L'étude de l'état physiologique des bourgeons entrés en repos à $25^{\circ} \mathrm{C}$ en jour long conduit à rechercher une des causes de la cessation d'activité au niveau des tissus sousjacents au bourgeon et même au méristème. Des analyses biochimiques indiquent que la régulation du métabolisme énergétique et des mécanismes de transports de nutriments (sucres, acides organiques, ions et hormones comme l'AIA ou l'ABA) sont impliqués dans l'inhibition de la croissance des bourgeons de certaines plantes pérennes (Gendraud, 1981 ; Lavarenne et al., 1982 et 1986 ; Gendraud et Lafleuriel, 1983 ; Barnola et al., 1986 (a) et (b)). Chez le Châtaignier, l'étude de la diffusion d'un acide faible (la 5,5'-diméthyloxazolidine 2,4-dione ou D.M.O.) indique que la capacité à croître d'un bourgeon est d'autant meilleure que l'acide s'accumule plus dans la "zone méristématique " du bourgeon. C'est le cas des bourgeons en croissance et des bourgeons du collet des plants cultivés à $25^{\circ} \mathrm{C}$ en jour long toujours aptes à croître. Au contraire, une forte accumulation de la D.M.O. est caractéristique des territoires adjacents aux bourgeons du collet de plants traités par le froid $\left(4^{\circ} \mathrm{C}\right)$ et devenus inaptes à la croissance (Pezet, 1983). Une étude préliminaire de la mobilisation des réserves amylacées et de la répartition des sucres simples solubles dans les bourgeons et l'axe sous-jacent permet d'envisager l'existence d'un cotransport $\mathrm{H}^{+}$-sucres au niveau du parenchyme ligneux dont la régulation modulerait les capacités de rétention des nutriments et serait un facteur de contrôle de l'aptitude à la croissance. 
Les conditions de culture, en particulier la température en modulant l'intensité des mécanismes physiologiques qui induisent et maintiennent la cessation d'activité du bourgeon (corrélations d'intensité et d'origines diverses qui conduisent à l'inertie partielle ou totale) modulent le rythme de croissance (amorti ou non) et la morphogenèse (sympode ou monopode, arbre ou buisson).

\section{Reçu le 20 février 1987. \\ Accepté le 20 juin 1987.}

\section{Summary}

Growth, morphogenesis and dynamics of bud growing ability of young Chestnut plants (Castanea sativa Miller) outdoors and under closely controlled conditions.

Young Chestnut plants exhibited an endogenous rythmic growth with alternate periods of elongation and inactivity under constant environmental conditions. The period of rest was irregular and varied with the increasing temperature.

In nature, Chestnut is a sympodial tree, but, under constant environmental conditions, it was either a sympodial bush or a typical monopodial tree. The falling apical bud and the decreasing correlative factors promoted the basal buds outgrowth under high temperature $\left(25^{\circ} \mathrm{C}\right.$ in long days). Whereas low temperature $\left(12^{\circ} \mathrm{C}\right.$ in long days) induced the formation of a scaly apical bud, prevented sprouting of basal buds and promoted the erection of a trunk.

The use of biochemical technic has been suggested to analyse changes of growth capacity buds according to their position along the stem in closely controlled environments.

Key words: Bud rest, Chestnut, morphogenesis, rhythm, temperature.

\section{Références bibliographiques}

Barnola P., 1976 (a). Recherches sur la croissance et la ramification du Noisetier (Corylus avellana L.). Ann. Sci. Nat., Bot., $12^{\mathrm{e}}$ série, 17 (3), 223-258.

BARNola P., 1976 (b). Recherches sur la dormance et la morphogenèse de quelques espèces ligneuses buissonnantes. Thèse Doct. ès Sci. Nat., Univ. Clermont-Ferrand II, 153 p.

Barnola P., Champagnat P., Lavarenne S., 1977. Mise en évidence d'une dormance rythmique chez le Noisetier (Corylus avellana L.) cultivé en conditions contrôlées. C. R. Acad. Sci., série D, 284, 745-748.

Barnola P., Lavarenne S., Gendraud M., 1986 (a). Dormance des bourgeons apicaux de Frêne (Fraxinus excelsior L.) : évaluation du pool des nucléosides triphosphates et éventail des températures actives sur le débourrement des bourgeons en période de dormance. Ann. Sci. For., 43 (3), 339-350.

Barnola P., Crochet A., Payan E., Gendraud M., Lavarenne S., 1986 (b). Modifications du métabolisme énergétique et de la perméabilité dans le bourgeon apical et l'axe sous-jacent au cours de l'arrêt de croissance momentané de jeunes plants de Chêne. Physiol. Vég., 24 (3), 307-314.

Bergougnoux F., Verlhac A., Breisch H., Chapa J., 1978. Le Châtaignier: production et culture. Invuflec Ed., Paris, $100 \mathrm{p}$.

Camus A., 1929. Les Châtaigniers. Paul Lechevalier Ed., Paris, 604 p. + Figures et cartes + Atlas.

Champagnat P., 1983. Quelques réflexions sur la dormance des bourgeons de végétaux ligneux. Physiol. Vég., 21, 607-618. 
Champagnat P., Barnola P., Lavarenne S., 1971. Premières recherches sur le déterminisme de l'acrotonie des végétaux ligneux. Ann. Sci. for., 28 (1), 5-22.

Champagnat P., Barnola P., Lavarenne S., 1986. Quelques modalités de la croissance rythmique endogène des tiges chez les végétaux ligneux. Colloque international sur l'Arbre. Naturalia monspeliensia, 279-302.

Crabré J., 1968. Evolution annuelle de la capacité intrinsèque de débourrement des bourgeons successifs de la pousse de l'année chez le Pommier et le Poirier. Bull. Soc. Roy. bot., Belgique, 101, 195-204.

De Chлмps I., 1972, La production des taillis de Chataignier. AFOCEL, 221-274 + Annexes.

Gendraud M., 1981. Etude de quelques propriétés des parenchymes des pousses de Topinambour cultivés in vitro en relation avec leurs potentialités morphogénétiques. Physiol. Vég., 19, 473481 .

Gendraud M., Lafleuriel J., 1983. Caractéristiques de l'absorption du saccharose et du tetraphenylphosphonium par les parenchymes de tubercules de Topinambour, dormants ou non dormants, cultivés in vitro. Physiol. Vég., 21, 1125-1133.

Guevara E., 1984. Croissance el morphogenèse du Pêcher-Amandier (Prunus persica x amygdalus) cv. GF-677 cultivé in vitro, puis en conditions phytotroniques diverses. Doc. Ing., ClermontFerrand II, $155 \mathrm{p}$. + Illustrations.

Hallé F., Martin R., 1968. Etude de la croissance rythmique chez l'Hévéa (Hevea brasiliensis Müll. Arg., Euphorbiacées-crotonoïdées). Adansonia, ser-2, 8, 475-503.

Hallé F. et Oldeman R.A.A., 1970. Essai sur larchitecture et la dynamique de croissance des arbres tropicaux. Collection de monographie de Botanique et de Biologie végétale, Masson et Cie Ed., Paris, 178 p.

HUET J., 1965. Les facteurs de l'induction florale. Les principales techniques à mettre en auvre pour régulariser les productions annuelles. Soc. Pomol. Fr., Congrès pomologique, $96^{\circ}$ session, $37-$ 50 .

Iivvuflec, 1975. Châtaignes et Marrons. Invuflec Ed., Paris, 148 p.

Jourdan J., 1980. Variations saisonnières de la morphogenèse et de la croissance des systèmes aériens et souterrains chez le Peuplier: leurs relations avec les glucides et les transferts minéraux. Thèse Doct. ès Sci. Nat., Univ. Sci. et Méd., Grenoble, 160 p. + Annexe.

LAMOND M., 1978. Influence des cotylédons sur la croissance et le développement du système racinaire du Chêne pédonculé (Quercus robur L.). C. R. Groupe d'étude des racines, Riedacker (A.) et Gagnaire-Michard (J.) éd., 5, 228-241.

Lavarenne S., Barnola P., Champagnat P., 1980. Climats artificiels et dormance des bourgeons. I - Températures et dormance automnale chez le Frêne (Fraxinus excelsior L.). C. R. Acad. Agric. Fr., (92-106).

Lavarenne S., Champagnat P., Barnola P., 1971. Croissance rythmique de quelques végétaux ligneux des régions tempérées cultivés en chambres climatisées à température élevée et constante et sous diverses photopériodes. Bull. Soc. Bot. Fr., 118, 131-162.

Lavarenne S., Champagnat P., Barnola P., 1975. Influence d'une gamme de températures sur l'entrée et la sortie de dormance des bourgeons du Frêne (Fraxinus excelsior L.). Physiol. Vég., 13 (2), 215-224.

Lavarenne S., Barnola P., Gendraud M. Jallut N., 1986. Caractérisation biochimique de la période de repos au cours de la croissance rythmique du Frêne cultivé à température élevée et constante. C. R. Acad. Sci., 303 (4), 139-144.

Lavarenne S., Champciaux M., Barnola P., Gendraud M., 1982. Métabolisme des nucléotides et dormance des bourgeons chez le Frêne. Physiol. Vég., 20, 371-376.

Levert J., 1982. Contribution à l'étude de la physiologie de la germination du Chêne pédonculé (Q. robur L.). Thèse $3^{\mathrm{e}}$ cycle, Clermont-Ferrand II, $163 \mathrm{p}$.

MAUgeT J.C., 1978. Influence d'une ablation totale du feuillage sur l'entrée en dormance des bourgeons du Noyer (Juglans regia L.). C. $R$. Acad. Sci., série D, 286, 745-748.

Mauget J.C., 1984. Comportement comparé des bourgeons de l'année et des bourgeons latents chez le Noyer (Juglans regia L. cv. «Franquette »). Conséquences sur la morphogenèse de larbre. Agronomie, 4 (6), 507-515. 
Meng-Horn C., Champagnat P., Barnola P., Lavarenne S., 1975. L'axe caulinaire, facteur de préséances entre bourgeons sur le rameau de l'année du Rhamnus frangula L. Physiol. Vég., 13 (3), 335-348.

PARISOT E., 1985. Etude de la croissance rythmique chez de jeunes manguiers (Manguifera indica L.). Thèse $3^{\mathrm{e}}$ cycle, Univ. Clermont-Ferrand II, $156 \mathrm{p}$.

Payan E., 1982. Contribution à l'étude de la croissance rythmique chez de jeunes Chênes pédonculés, Quercus pedunculata Ehrh. Thèse $3^{i}$ cycle, Clermont-Ferrand II, 102 p.

PEzET Y., 1983. Contribution à l'étude de quelques propriétés des bourgeons de Châtaignier (Castanca sativa Miller): Absorption de la D.M.O. et du tetraphenylphosphonium. D.E.A., Clermont-Ferrand II, 55 p. + Illustrations.

POUGEt R., 1963. Recherches physiologiques sur le repos végétatif de la Vigne (Vitis vinifera $L$.) : la dormance des bourgeons et le mécanisme de sa disparition. Thèse Doct. Sci. Nat., Bordeaux.

Rageau R., 1975. Problèmes posés par l'évaluation quantitative de la dormance. Exemples pris chez le Pêcher. Groupe d'étude de la physiologie de l'arbre, 8 p.

Regnard J.L., 1984. Rôle de l'Anoxie dans la levée de Dormance des bourgeons de Peuplier (Populus x euramericana (Dode) Guinier, cv. I 214) : Analyse des Processus physiologiques. Thèse $3^{c}$ cycle, Paris VI, $151 \mathrm{p}$.

Riedacker A., 1981. Croissance aérienne et souterraine. In : Le Hêtre, I.N.R.A. Paris, 160-169.

Si-Moнамеd C., 1983. Germination; rythmes de croissance et morphogenèse de jeunes plants chez Castanea sativa Miller. Thèse $3^{\mathrm{e}}$ cycle, Univ. Clermont-Ferrand II, 201 p. + Illustrations.

Vegis A., 1964. Dormancy of higher plants. Ann. Rev. Plant Physiol, 15, 185-224.

Vogel M., 1975. Croissance rythmique du Cacaoyer. Thèse $3^{c}$ cycle, Paris sud, $88 \mathrm{p}$.

ZANETTE F., 1981. Recherches descriptives et expérimentales sur la morphogenèse des systèmes aériens et racinaires de quelques porte-greffes de Pommier. Thèse Doc. Ing., Univ. ClermontFerrand II, 159 p. + Illustrations. 